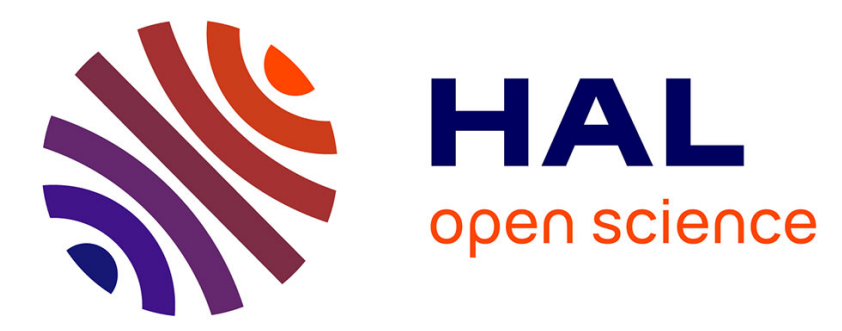

\title{
Euvres et pratique du jeûne dans les Eglises réformées de France (1650-1750) : principes, contestations et évolutions
}

Didier Boisson

\section{- To cite this version:}

Didier Boisson. (Euvres et pratique du jeûne dans les Eglises réformées de France (1650-1750): principes, contestations et évolutions. Céline Borello. Les oeuvres protestantes en Europe, Presses universitaires de Rennes, pp.179-191, 2013, 978-2-7535-2811-6. hal-03431123

HAL Id: hal-03431123

https://univ-angers.hal.science/hal-03431123

Submitted on 16 Nov 2021

HAL is a multi-disciplinary open access archive for the deposit and dissemination of scientific research documents, whether they are published or not. The documents may come from teaching and research institutions in France or abroad, or from public or private research centers.
L'archive ouverte pluridisciplinaire HAL, est destinée au dépôt et à la diffusion de documents scientifiques de niveau recherche, publiés ou non, émanant des établissements d'enseignement et de recherche français ou étrangers, des laboratoires publics ou privés. 


\title{
Chapitre $X$ \\ Cuvres et pratique du jeûne dans les Églises réformées de France (1650-1750) : principes, contestations et évolutions
}

\author{
Didier BoIsson
}

\begin{abstract}
"La divine providence ayant permis le renouvellement de la persécution dans plusieurs provinces de ce royaume pour punir son Eglise de l'abus qu'elle avait fait des grâces dont le Ciel l'avait favorisée les années précédentes, nous avons vu avec la douleur la plus vive la désolation de nos confrères, l'emprisonnement des uns, la condamnation des autres et la suppression de nos exercices religieux. Réfléchissant sur nos maux présents et à venir, et considérant que la multiplication de nos iniquités est un obstacle insurmontable à la délivrance après laquelle nous soupirons depuis longtemps, nous avons résolu de célébrer un jeûne solennel le jeudi 18 août prochain..., jour auquel tous les fidèles commis à nos soins sont exhortés à s'humilier extraordinairement devant Dieu, à se repentir sincèrement de leurs crimes, à prier Dieu pour la paix de l'Europe et de l'Église, et aussi bien pour la prospérité de Sa Majesté, notre auguste monarque. Et afin de donner à cette solennité l'éclat que nous désirons, on s'abstiendra autant qu'il leur sera possible de toute œuvre servile le jour marqué pou vaquer entièrement au service divin ${ }^{1}$. "
\end{abstract}

Voici une formulation quasiment intemporelle par laquelle un synode demande la célébration d'un jeûne. Selon la Discipline ecclésiastique, « en temps d'âpre persécution, ou de peste, ou de guerre, ou de famine, ou autre grande affliction. Item quand on voudra élire les ministres de la parole de Dieu, et quand il sera question d'entrer au synode, on pourra si la nécessité le requiert, à certain jour, ou plusieurs, dénoncer les prières publiques et

1. Hugues E., Les synodes du Désert, Paris, 1886, vol. 1, p.224. Art. 1 du synode provincial de Haut-Languedoc (1746). 
extraordinaires, avec le jeûne, toutefois sans scrupule et superstition, le tout avec grande cause et considération. Et les Églises sont averties de se conformer les unes aux autres en la célébration du jeûne, autant que faire se pourra, selon la commodité des temps ${ }^{2}$ ". Si on saisit aisément les circonstances dans lesquelles un jeûne peut être célébré, on ne peut pas dire que la Discipline ecclésiastique soit très explicite sur les objectifs de cet exercice sacré. En quoi le jeûne, pratique communautaire, rite collectif au cours duquel les membres de l'Église passent leur journée à prier, à chanter des psaumes, à écouter des sermons et à s'abstenir de boire et de manger, peut-il être considéré comme un secours spirituel, une entraide spirituelle dans une Église réformée 3 ? Peut-on observer une évolution dans cette conception du jeûne dans les décennies qui précèdent la révocation de l'édit de Nantes et le temps du Désert? Pour étudier le jeûne comme forme d'œuvre dans les Églises réformées de France, on peut s'appuyer à la fois sur des sources institutionnelles (actes de synodes nationaux, actes de synodes provinciaux, actes de synodes du Désert), sur des sermons de pasteurs qui ont été prêchés au cours de ces années et sur des ouvrages de controverse. Une des difficultés rencontrées est que les études sur le jeûne sont peu nombreuses. Ce sont tout d'abord les pages que Paul de Félice a consacrées à cette pratique cultuelle ${ }^{4}$. Raymond Mentzer a publié récemment un article au jeûne dans lequel il procède à une analyse plus anthropologique et beaucaun moins descriptive que Paul de Félice $^{5}$. Ainsi, trois points peuvent être é és: tout d'abord le jeûne comme élément de cohésion des communautés, puis le jeûne comme objet de division des communautés, enfin l'évolution que l'on peut observer avec les Églises du Désert dans la première moitié du XvıII ${ }^{\mathrm{e}}$ siècle.

Face aux persécutions, le jeûne apporte un secours spirituel, permet de souder la communauté et de maintenir le lien social entre ses membres, mais aussi avec les autres communautés du royaume ou de l'étranger. La conversion engendrée par le jeûne doit être collective et non individuelle: c'est ce qu'exprime Fabrice Burlamaqui, pasteur de Grenoble, dans un sermon prononcé en $1663^{6}$. Le vocabulaire utilisé par les ministres cherchent à

2. D’Huisseau I., La Discipline des Églises réformées de France, Saumur, 1666, chap.X, art. 3.

3. Mentzer R., "Fasting, piety and political anxietry among french reformed protestants ", Church History, 76 (2007), p. 330-362.

4. Felice P. de, Les protestants d'autrefois. Les temples. Les services religieux. Les actes pastoraux, Paris, Librairie Fischbacher, 1896, p. 154-171.

5. Mentzer R., op. cit. Voir également les pages consacrées au jeûne dans Grosse C., Chevalier F., Mentzer R., Roussel B., "Anthropologie historique: les rituels réformés (Xvie-XVII ${ }^{\mathrm{e}}$ siècles)", Bulletin de la Société de l'Histoire du Protestantisme Français, t. 148, octobre-décembre 2002, p. 979-1009.

6. Sermon fait au jour de jusne celebre par les eglises reformees de Daufiné, le 3. jour de Decembre 1662, par Fabrice Burlamachi, ministre de l'eglise reformee de Grenoble, Genève, 1663. Il écrit ainsi: "Nous devons prendre tellement garde à la voix de ses châtiments, qu'elle nous ouvre l'oreille et nous porte à écouter plus soigneusement les enseignements de sa bouche; qu'elle nous forme à les recevoir avec tant de soumission que nous passions à des résolutions plus fortes que nous n’avons eu contre le péché, que nous reprenions le vrai zèle évangélique au lieu des relachements qui font méconnaitre le 
souligner ce changement de comportement collectif dû au jeûne. Ainsi, dans un sermon prononcé par Jean Claude à La Haye en novembre 1685, l'auteur insiste, naturellement dans le contexte particulier de la révocation de l'édit de Nantes, sur une opposition entre d'un côté les malheurs, l'affliction, les calamités, la douleur, les maux, les ténèbres, et de l'autre, la joie, les lumières, la paix, la consolation ou le triomphe que doit apporter le jeûne ${ }^{7}$. Dix ans plus tôt, dans un autre sermon à l'occasion de la célébration d'un jeûne, le même Jean Claude a déjà évoqué les sentiments que doit avoir le réformé lors d'un tel culte ${ }^{8}$. Le pasteur qualifie le Dieu « devant qui nous sommes humiliés ", comme un Dieu « juste ", un Dieu " vengeur », "père de miséricorde ", "père d'espérance 9 ". Ainsi, par le jeûne, "le premier fruit que nous devons attendre de notre repentance et de notre humilité, c’est le pardon de nos péchés ${ }^{10}$ ", mais " Dieu ne pardonne les péchés qu’à ceux qui ont revêtu un esprit de gratuité et de vérité. Il faut être bon, miséricordieux et charitable envers nos frères si nous voulons que Dieu le soit envers nous ${ }^{11}$ ". À partir du verset des Proverbes Quant l'Eternel prend plaisir aux voies de l'homme, il apaise même envers lui ses ennemis ${ }^{12}$, Claude définit ainsi les "voyes " de Dieu et celles des hommes:

"Les voies de l'homme sont ses mouvemens, ses sentimens, ses pensées, ses paroles, ses actions, en un mot toute sa conduite. L'Écriture nous parle de deux sortes de voyes, celles de Dieu et celles de l'homme. Celles de Dieu sont les voyes de sa providence, par laquelle il gouverne en général toutes les créatures, et en particulier son Église. Celles de l'homme sont les œuvres et les manières de sa vie ${ }^{13}$."

Ce sermon de Jean Claude permet de comprendre comment les comportements des hommes lient œuvres et jeûne. Ainsi, « les voyes de Dieu sont cachées à nos yeux, elles ne nous paraissent que par les événements, c'està-dire par les vestiges et les impressions que Dieu y laisse quand il y passe, mais d'elles mêmes, elles nous sont inconnues ". En revanche, "celles de l'homme sont toujours exposées aux yeux de Dieu, il les voit avant que l'homme y entre, il les contemple quand il y est, et il s'en souvient quand il est passé ${ }^{14} »$. En effet:

christianisme au milieu de nous, afin de montrer à la suite en notre vie cette belle pureté que nous avons dans la doctrine» (p. 11).

7. Claude J., Sermon sur le verset 14 du chapitre 7 de l'Ecclésiaste, prononcé à La Haye le 21 novembre 1685, jour de jeûne, Londres, 1686.

8. Claude J., Les fruits de la repentance ou sermon sur ces paroles de Salomon il y aura propitiation pour l'iniquité par gratuité et vérité..., prononcé à Charenton le 3 avril 1676, jour de jeusne, Charenton, 1676.

9. Ibid., p. 1-2.

10. Ibid., p. 6.

11. Ibid., p. 12 .

12. Proverbes 16, 7.

13. Claude J., Les fruits de la repentance, op. cit., p. 19.

14. Ibid. 
"Les voyes de Dieu sont partout, tout l'univers est son chemin, car toutes choses sont soumises à l'ordre de sa providence. Celles de l'homme sont étroites, bornées et de fort petite étendue. Celles de Dieu sont justes et droites... Les voyes de l'homme sont quelquefois droites, mais quelquefois aussi ce ne sont que des égarements. Nos voyes sont droites quand nous suivons notre vocation et que nous obéissons à la Loy de Dieu. Ce sont au contraire des égaremens quand nous nous écartons de notre vocation et que chacun de nous veut être et son guide et son flambeau ${ }^{15}$."

Par le jeûne, l'ensemble de la communauté doit suivre une nouvelle attitude, le jeûne est bien un temps de conversion personnelle, mais surtout collective. C'est donc dans cet objectif qu'un jeûne national ou provincial doit être entendu, le lien social peut être bien au-delà des membres de la communauté et s'étendre à des Églises réformées de l'étranger. Une Église qui n'organise pas le jeûne public en même temps que les autres, se voit censurée. C'est ce qui arrive par exemple à celle de Châteaudun pour avoir célébré le jeûne le 6 novembre, au lieu du 5 novembre, jour fixé par le synode national de Saint-Maixent de $1609^{16}$. Le jeûne du vendredi saint de l'année 1658, célébré à Charenton le 19 avril, est bien connu grâce à de nombreuses sources. L'Acte pour la célébration du jềne, qui a été lu au temple de Charenton les 7 et 14 avril, évoque « une force ennemie qui [...] a fait tant de ravages dans les Église semées çà et là en divers pays qui en a ébranlé les unes et dissipé les autres, employant en quelques lieux la violence et l'horreur de la cruauté et en d'autres la fraude et les artifices pour ruiner les sanctuaires du Seigneur ${ }^{17}$ "; de ce jeûne nous possédons également l'ordre pour le culte ${ }^{18}$ et le texte des trois sermons prêchés ce jour-là, ceux de Jean Daillé, puis de Raymond Gaches et enfin de Charles Drelincourt ${ }^{19}$. Un des témoins, Philippe de Villers, écrit dans son Journal:

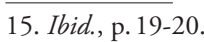

16. Felice P. de, Les protestants d'autrefois, op. cit., p. 157. Voir art. XIII des Matières générales du synode national de Saint-Maixent: "Qu’attendu le mépris de la parole de Dieu, les blasphèmes, les débordemens et dissolutions qui se trouvent en plusieurs lieux, \& en somme le défaut de loi et de charité qui n'est que trop commun et qui nous menace de l'ire de Dieu, attirée par l'impiété et l'injustice des hommes, il seroit à propos de célébrer un jeûne public par toutes les Églises de ce royaume pour s'humilier extraordinairement devant Dieu et prévenir ses jugemens".

17. Actes pour la célébration du jusne qui a esté célébré à Charenton le vendredy 19. d'avril 1658. Ce document comprend en particulier une Exhortation au jusne et à la repentance avec des prières pour demander à Dieu la sanctification du jûne et la rémission des péchez, Charenton, 1658.

18. Ordre qui a esté donné au lecteur pour ce qui devoit estre leu et chanté le jour de jûne célébréà Charenton le 19. d'avril 1658, dans Actes pour la célébration, op. cit.

19. DAIllÉ J., Sermon sur l'Apocalypse, chap. 11, vers. 5, prononcé à Charenton le vendredy 19 d'avril 1658, jour de jeusne, Paris, Samuel Perier, 1658; Gaches R., Sermon sur Jeremie, chap. III, v. 22, prononcé à Charenton le vendredy 19 d'avril 1658, jour de jeusne, Paris, Samuel Perier, 1658; Drelincourt C., La repentance de l'enfant prodigue ou sermon sur le XV de Saint Luc depuis le verset 11 jusques au 24, prononcé à Charenton le vendredy 19 avril, jour de la célébration du jûne, Paris, Antoine Cellier, 1658. De nombreux autres sermons prononcés lors de jeûnes ont été publiés. On peut citer par exemple: Drelincourt C., Sermon pour le jusne celébré à Charenton pour la prosperité des armes du Roy, le jeudy 21 aoust 1636, Rouen, 1636; Le FAucheur M., Exhortation à la repentance, faite en l'Église de Montpellier, pour la sanctification du jeusne, célébré par les Églises de France, le dixiesme d'octobre 
"Le $19^{\mathrm{e}}$ les grands efforts du clergé contre nos églises, la corruption de nos mœurs et le peu de zèle qui règne parmi nous, faisant appréhender à ceux de notre religion qui sont en cette ville que Dieu ne voulut nous châtier de notre impiété et irréligion en nous ôtant la prédication de sa parole et nous punissant de la façon que le furent les églises d'Asie dont parle saint Jean, leur firent choisir ce jour pour l'employer au jeûne et à des prières extraordinaires. Nous fûmes à Charenton depuis les huit heures du matin jusqu'aux six heures de l'après dînée. Nous y ouïmes trois beaux prêches et fort touchants, et Dieu veuille que nous en ayons bien fait notre profit $^{20}$."

Il retourne à Charenton le 21 avril pour la célébration de la Cène. Il écrit alors :

" [Nous] y fûmes aussi longtemps que le vendredi sans boire ni manger: et il faut avouer que le jeûne est une grande aide pour la piété et pour la dévotion ${ }^{21}$."

Ainsi, l'exemple de ce jeûne de 1658 montre également que cette unité du corps social est symbolisée par une unité de lieu pour la célébration, le temple.

Fixé par les synodes nationaux, mais aussi dans la seconde moitié du $\mathrm{XVII}^{\mathrm{e}}$ siècle plus particulièrement par les synodes provinciaux, les jours de jeûnes n'ont pas de périodicité régulière, à la grande différence naturellement du jeûne catholique. Si les synodes nationaux décident communément d'un jour de jeûne - c'est le cas jusqu'au dernier synode national de Loudun de 1659, nous y reviendrons -, il n'en est pas de même des synodes provinciaux. L'exemple de la province d'Anjou-Touraine-Maine est à cet égard éclairant: les députés n'ordonnent pas la célébration de jeûnes publics à la fin du XVI ${ }^{\mathrm{e}}$ siècle ou pendant la première moitié du XVII ${ }^{\mathrm{e}}$ siècle, ce sont les synodes nationaux qui s'en chargent. À partir des années 1650, cette règle évolue. Le jeûne de 1658, sans être décrété par un synode national, est célébré dans de nombreuses provinces, mais à des dates différentes, par exemple en avril pour l'Île-de-France-Champagne-Picardie, en novembre pour l'Anjou-Touraine-Maine ${ }^{22}$. Dans cette même province, un autre jeûne

1618, Montpellier, 1618; JANSSE L., Les larmes chrétiennes, ou sermons faits en des jours de jeusne, Rouen, 1669.

20. Villers P. de, Journal d'un voyage à Paris en 1657-1658, Paris, 1862, p. 459.

21. Ibid., p. 461. La question de l'abstinence de nourriture est peu évoquée par les réformés quand il s'agit de jeûne, d'où les provocations lancées à l'égard des catholiques par certains protestants pendant le carême et en particulier le vendredi saint. Après la révocation de l'édit de Nantes, refuser le jeûne catholique devient même un signe de résistance et d'opiniâtreté.

22. Actes des synodes provinciaux des Églises réformées d'Anjou-Touraine-Maine, édition présentée et annotée par Didier Boisson, synode provincial de Preuilly (1658), à paraitre. Voir article 1 des faits généraux: «Il a esté proposé a cette compagnye par les deputez de Loudun que le sinode nationnal ayant esté differé jusques a cette heure et n'y ayant pas aparance de l'obtenir sy tot, il seroit a propos pour beaucoup de raisons importantes de suivre un jeusne g[ene]ral dans toutes les eglizes du royaume selon que les reiglements sy donnent l'authorité aux provinces et aux eglizes quy ont droict 
est célébré en 1683. Même si plusieurs actes de synodes provinciaux manquent pour les années 1660, on constate que cette institution ne cherche pas à rendre la célébration des jeûnes d'une façon plus régulière, même dans un contexte de plus forte persécution durant le règne personnel de Louis XIV. Cependant, le jeûne de 1683 insiste sur la nécessité de maintenir le lien social entre les fidèles des différentes communautés, alors que la répression sévit avec plus d'intensité et que les conversions au catholicisme se multiplient ${ }^{23}$. Il est demandé aux fidèles:

«De garder en touttes leurs actions la modestie chretienne surtout de vuider leurs cœurs du vieux levain d'animosité pour ne pas deschirer par leurs divisions le corps mistique du fils de Dieu, mais esteindre plustost par une vehemente charité, l'union de l'esprit par le lien de la paix pour n'estre tous ensemble qu'un mesme cœur, et pour cet effet elle enjoint aux consistoires de travailler puissamment a reconcilier les membres de leur troupeau qui sont en division jusques a susprendre de la communion de la $s^{\text {te }}$ cene ceux qui se trouveront refractaires aux admonitions qui leur seront faites sur ce sujet ${ }^{24}$. »

Dans le contexte particulier de ces années qui précèdent la révocation de l'édit de Nantes, le jeûne peut apparaître également comme "à décourager toute rébellion contre une autorité répressive ${ }^{25}$ ", à l'image de ce qui se passe également en Normandie comme a pu l'étudier Luc Daireaux ${ }^{26}$. Ainsi, le jeûne réformé est un élément " régulé et ritualisé » du culte réformé et apparaît « comme un bel exemple du réemploi d'une discipline séculaire au profit d'objectifs renouvelés: intercession, apaisement. Public et imposé à la communauté, il protège le lien social qui y a été établi : la mise en avant du péché de tous occulte l'enquête sur d'éventuelles responsabilités particulières, les longues dévotions détournent de la panique apocalyptique, la nécessité d'être ensemble fait obstacle à la formation éventuelle de groupuscules activistes ${ }^{27}$."

de la convocation du sinode nationnal, sur quoy a esté arresté que plusieurs raisons tres considerables prises de la conjecture des temps et des affaires, et veu mesme que le jeusne a esté desja celebré dans plusieurs provinces et eglizes de ce royaume, on en doibt differer l'induction generalle et en aultre temps qu'il sera jugé plus a propos et plus convenable pour une chose de telle matiere ayant au prealable pris l'avis des eglizes de Paris, Tours, La Rochelle et autres ausquelles on escrira des memoires pour les consulter dans une affaire en cette importance ".

23. C'est au cours de ce synode provincial qui a eu lieu à Sorges, près d'Angers, que les députés des différentes Églises doivent accepter, à la demande du commissaire catholique, la présence des deux pasteurs de Baugé et de Château-du-Loir venus annoncer leur conversion prochaine au catholicisme.

24. Actes des synodes provinciaux des Églises réformées d'Anjou-Touraine-Maine, synode provincial de Sorges (1683).

25. Grosse C., Chevalier F., Mentzer R., Roussel B., "Anthropologie historique, op. cit. », p. 998.

26. Daireaux L., "Réduire les huguenots". Protestants et pouvoirs en Normandie au XVII siècle, Paris, Honoré Champion, 2010, p. 598-600. La célébration de jeûnes ordonnés par le synode provincial de Normandie apparaît plus régulière dans cette période. Ainsi les assemblées de 1675, de 1679 et de 1682 en font la demande.

27. Grosse C., Chevalier F., Mentzer R., Roussel B., «Anthropologie historique, op. cit. », p. 998. 
Mais le jeûne, dans cette analyse-là, ne peut-il pas être un thème de controverse entre réformés et catholiques, dans la mesure où il serait analysé comme un élément de division du corps social? En mars 1660, dans une lettre envoyée aux pasteurs et anciens de l'Église de Poitiers, Samuel Cottiby prend prétexte du jeûne décidé par le synode national de Loudun pour annoncer sa conversion au catholicisme ${ }^{28}$. Ce jeûne doit être célébré dans toutes les Églises réformées de France le jeudi 25 mars, le jeudi de la semaine sainte, et c'est justement ce 25 mars qu'il abjure entre les mains de l'évêque de Poitiers. Les actes du synode national de Loudun expliquent:

«Bien qu'il ait plu à Dieu de faire la grâce à notre souverain de terminer une longue et funeste guerre par une glorieuse paix [...], et ainsi de présenter à tous les gens de bien un ample sujet d'une joye universelle, néanmoins nous ne laissons pas de voir avec effroi et douleur que l'athéisme, l'impiété, le blasphème, l'injustice, la dissolution, le luxe et autres semblables péchés se vont multipliant et marchent la tête levée comme si les hommes avaient entrepris de provoquer avec fierté la justice de Dieu et embraser de plus en plus le feu de sa dernière colère ${ }^{29}$."

Ce jeûne a été l'objet de discussions et de réserves dans les années précédant le synode national de Loudun, comme le montre cet article du synode provincial d'Anjou-Touraine-Maine de 1656: un pasteur de Loudun y présente:

« une lettre de l'eglise de Grenoble par laquelle lad. eglise de Lodun estoit exhortée d'induire un jeusne general pour toutes les eglises de ce royaume, on a esté d'advis qu'en considera[ti] on de la hayne de nos adversaires et des temps qu'on n'estime pas propres pour l'indiction d'un tel jeusne, on disfairoit de le faire jusques au temps qu'il plaira a Sa Maté de permettre la tenue du sinode nationnal ${ }^{30}$ ".

Dans la lettre où il annonce sa conversion, Cottiby définit ainsi le jeûne:

« Je scay bien que le jeusne est louable et necesseaire, qu'il est pour les maladies de nos ames, ce qu'est un baume excellent pour les blessures de nos corps; je scay que c'est par le jeusne que le Ciel est desarmé, et la colère de Dieu appaisée; avec cette sainte abstinence jointe a la priere, on arreste ses jugemens et on attire ses benedictions ${ }^{31}$."

28. Deux lettres de Mr Cottiby ministre de Poictiers, à mademoiselle Cottiby sa mère, et à un ecclésiastique de ses amis, sur le sujet de sa conversion à la foy catholique, Paris, 1660. Son père, Jacques Cottiby, également ancien pasteur de Poitiers, s'était converti au calvinisme et avait été délégué de la province de Poitou au synode national de Charenton de 1623.

29. Aymon J. Tous les synode nationaux des Églises réformées de France..., La Haye, 1710, vol. II.

30. Actes des synodes provinciaux des Églises réformées d'Anjou-Touraine-Maine, synode provincial de Baugé (1656), art. 4.

31. Lettre du sieur Cottiby, cy-devant pasteur de l'Eglise réformée de Poitiers, envoyée au consistoire de ladite Eglise, avec les responces à sa lettre, Charenton, 1660, non paginée. 
Ce qu'il conteste, c'est justement le fait que le jeûne soit considéré par les réformés comme un temps d'entraide spirituelle entre réformés et qu'il soit célébré "à contre temps, au grand scandale de toute la France " par un seul corps de la société. Élément d'unité des communautés réformées, il devient un élément de division entre sujets du souverain. Le jeûne est considéré par Cottiby comme un élément d'opposition politique:

" Je vois bien, écrit-il, que c'est l'accord et l'union des deux plus grandes puissances de l'Europe, qui fait pour vous cette constellation maligne que vous prenez à mauvais augure. Et c'est ce qui gesne d'une étrange manière les esprits de tous les véritables François, qu'il leur faille par un motif de religion avoir des interests séparez de ceux de leur prince naturel et de leur chère patrie, faisans notre amertume de leur douceur, nostre nuit de leur jour, de leur lumière nos ténèbres ${ }^{32}$."

Cette conversion a naturellement entraîné plusieurs réactions protestantes. La première est publiée dès 1660 à Paris ${ }^{33}$. Ouvrage écrit rapidement comme une première réponse au changement de confession du pasteur, il se veut tout d'abord ironique sur les raisons qui auraient conduit Cottiby à la conversion: en effet, comment condamner les réformées de jeûner un jeudi saint, sachant que les catholiques jeûnent ce même jour et les trente-neuf autres jours du carême ${ }^{34}$ ? L'auteur s'insurge ensuite contre l'interprétation politique faite au jeûne, soulignant combien « ceux de notre Religion en tous les lieux de leurs habitations ont été les premiers, ou du moins n'ont pas été les derniers, à prendre part aux feus de joie ${ }^{35}$ ». Il réfute ainsi l'accusation que le jeûne "s'est fait contre la paix », soulignant que des actions de grâce avaient été publiées dans plusieurs Églises réformées comme Saumur, La Rochelle et Sedan, avant même le jeûne, pour célébrer la paix ${ }^{36}$. La controverse est aussi un moyen de contester le carême en citant saint Augustin:

32. Lettre du sieur Cottiby, cy-devant pasteur de l'Eglise réformée de Poitiers, envoyée au consistoire, op. cit. Il est intéressant de noter que le synode provincial de Normandie prévoit la célébration d'un jeûne en 1679, mais il a ensuite décidé de la différer. Voir la lettre de Pierre Bayle à son frère Jacob : «On a trouvé fort étrange que pendant que toute la France se réjouissoit de la paix que le roy a faitte aux conditions qu'il luy a plu, nous fissions des jeûnes comme pour marquer que la félicité publique de l'État est regardée parmi nous comme le plus grand fléau de nos Églises ", texte publié par Labrousse E., McKenna A., Correspondance de Pierre Bayle, Oxford, Voltaire Foundation, 2003, t. 3 , p. 175 .

33. Lettre du sieur Cottiby, cy-devant pasteur de l'Église réformée de Poitiers... avec les responces à sa lettre, Paris, Louis de Vendosme, 1660. L'ouvrage contient en particulier une Lettre de Monsieur Le Coq au sieur de La Talonnière sur le changement de religion de M. Cottiby.

34. Ibid., p. 6. «Il commence par un reproche qu’il nous fait du jeusne que nous célébrasmes le jour de son abjuration. Fut-il jamais une plainte plus extravagante? Ceux de la commuunion où il s'est jetté nous accusent d'abolir les jeusnes. Celuy-ci sort d'avecq nous car nous jeusnons. Il ne peut souffrir que nous jeusnions un jour, et il se joint à ceux qui alors mesme en jeusnoient quarante tous d'une suite, sans en conter quelques autres [...]. "

35. Ibid., p. 10-11.

36. Ibid., p. 10. 
" Je vois bien, dit-il à Casulanus, que le jeusme est recommandé par le Nouveau Testament, mais je ne vois point défini par le commandement du Seigneur ou des apôtres à quels jours il faut jeûner et à quels jours il ne le faut pas $^{37}$."

Deux ans plus tard, Jean Daillé publie une Réplique aux ouvrages catholiques parus au moment de la conversion de Samuel Cottiby ${ }^{38}$. Daillé adresse cette réponse à la communauté de Poitiers en raison de la " douleur " " causée par l'affliction que vous donna il y a deux ans le changement de l'un de vos conducteurs ${ }^{39}$ ». En plus de neuf cents pages, le ministre de Charenton répond ainsi aux accusations de Cottiby, mais pas seulement: c'est un vrai traité de théologie réfutant toutes les accusations classiques exprimées par les catholiques contre le calvinisme et dénonçant en sens inverse les erreurs et superstitions catholiques ${ }^{40}$. À l'égard de ceux qui estiment que les protestants, à travers le jeûne, rompent l'unité de la société et de la chrétienté, Daillé utilise des arguments similaires à ceux développés déjà en 1660. Il revient sur la fidélité des protestants à l'égard du souverain, en particulier ceux des villes de Saumur, La Rochelle et Sedan, expliquant ainsi que la ville dont Duplessis-Mornay avait été le gouverneur avait été toujours fidèle, de même que Sedan depuis sont rattachement et que La Rochelle avait été pardonnée. On ne peut donc pas considérer que les communautés de ces trois villes aient cherché à rompre l'unité du royaume au moment de la paix de 1659 et du mariage du roi ${ }^{41}$. D'autant que Cottiby, en écho aux événements qui se sont déroulés en Angleterre, accuse parallèlement les protestants de détrôner les souverains et de les faire exécuter ${ }^{42}$. Daillé refuse naturellement cette assimilation, même si la Révolution anglaise a divisé les réformés français ${ }^{43}$. Le thème du jeûne apparaît donc comme un élément de la controverse, non pas uniquement

37. Lettre du sieur Cottiby, cy-devant pasteur de l'Église réformée de Poitiers... Response à la lettre du sieur Cottiby, op. cit., p. 19.

38. Réplique de Jean Daillé aux deux livres que messieurs Adam et Cottiby ont publiés contre luy, Genève, 1662, 428 p. Une nouvelle édition parait en 1669, revue et corrigée, comprenant désormais plus de 800 p. C'est cette seconde édition que j'utilise.

39. Ibid., épître.

40. C'est ainsi que sont évoqués par exemple la transsubstantiation, l'intercession des saints, la confession auriculaire, le culte des images...

41. Réplique de Jean Daillé, op. cit... III De l'innocence de notre Religion, p. 119-120. "Car puisque nous avons eu toute la joye que de bons sujets devoyent avoir pour la paix, pour le mariage, et pour la prospérité du Roy, il est clair que cela n’a pu avoir esté le sujet de notre deuil, c'est-à-dire de notre jeusne et de notre affliction devant Dieu ".

42. Ibid., p. 124. "Mais de quel droit? Quelle part y avons-nous eue? \& pourquoy sommes-nous responsables des fureurs de quelques étrangers contre leur prince? Avons-nous été dans leur Conseil? Avons-nous approuvé leur parricide? Mais qui s'est écrié plus haut que nous contre leur impiété barbare et dénaturée? M. de Saumaise... »

43. Sur cette question, voir le conflit qui oppose à la fin des années 1650 la communauté de Saumur et en particulier les pasteurs Amyraut et d'Huisseau. Chevalier F., « La contestation de l'autorité du pasteur par l'assemblée des chefs de famille: Saumur, 1655-1660 ", L'anticléricalisme intraprotestant en Europe continentale (XVII -XVIII siècles), textes réunis par Yves Krumenacker, Lyon, Institut d'Histoire du Christianisme, université de Lyon 3, 2003, p. 11-26. 
sur des motivations théologiques, mais aussi pour montrer que les protestants par cette pratique rompent l'unité du royaume, ce qui permet de mettre en doute leur loyalisme à l'égard du roi.

Le temps du Désert ne modifie pas l'approche du jeûne comme secours spirituel et élément permettant de souder la communauté. Cette caractéristique s'observe dès le temps des persécutions précédant la révocation de l'édit de Nantes. Le projet de Claude Brousson de 1683 le montre aisément. Ainsi, « le dimanche 4 juillet prochain, toutes les églises célèbreront moyennant la grâce de Dieu un jeûne solennel. On commencera par une prédication sur la conversion à Dieu et la réformation des mours, à quoi tous les sujets seront exhortés d'une façon extraordinaire ${ }^{44}$ ». Plusieurs prières doivent alors être faites:

"La première pour la confession générale de nos péchés. La seconde pour implorer la grâce et le salutaire secours du Saint-Esprit, pour la régénération de nous et de nos frères et pour le continuel accroissement de nos sanctifications. La troisième pour implorer la protection du ciel sur nous et sur nos frères, dans le déplorable état où nous sommes tous réduits. La quatrième pour demander à Dieu le zèle et la fermeté nécessaire à nous et nos frères pour lui donner gloire et pour édifier tous les peuples du monde. Mais ce modèle est plutôt dressé pour unir nos vœux que pour restreindre les mouvemens de piété des pasteurs ${ }^{45}$."

Le jeûne appartient sans aucun doute à cette " ecclésiologie de crise ", si l'on reprend une expression d'Hubert Bost ${ }^{46}$. La nécessité de fuir loin des lieux habités, exprimée ainsi avant la Révocation par Brousson, s'accompagne par le jeûne d'un renforcement de cette idée d'apporter un secours spirituel. Le jeûne est alors un élément qui permet d'entretenir la conscience martyre après 1685 et d'élaborer un contre-modèle clandestin ${ }^{47}$.

Ainsi on ne peut être que frappé par la continuelle organisation de jeûnes dans les synodes provinciaux et nationaux du Désert, beaucoup plus

44. Brousson C., Apologie du Projet des réformés fait au mois de may 1683... contenant la suite de l'état des reformez où l'on apporte les traitemens que souffrent encore ceux qui se sont assemblez pour prier 45. Idem

Dieu..., Cologne, Pierre Marteau, 1684, p. 106-107.

46. Bost H., "Clause Brousson et le Désert des huguenots: une poétique de l'épreuve ", Ces Messieurs de la RPR, Paris, Honoré Champion, 2001, p. 237-265. Il faut bien souligner que si le projet de Claude Brousson échoue, il met en lumière l'opposition qui est vivement ressentie entre protestantisme urbain et aisé du Nord du royaume et un protestantisme méridional rural et plus populaire, comme l'exprime l'Apologie pour les protestants du Dauphiné: « Nous faisons une remarque nous autres protestans qui habitons la campagne, que la plupart de vous autres, Messieurs les protestants des grandes villes, vous avéz un zele fort languissant pour le service de Dieu... ", texte publié par Labrousse É., Avertissement aux protestants des provinces, Paris, 1986, p. 47.

47. Hubert Bost souligne que chez certains pasteurs du Refuge, comme Pierre Jurieu, il y a très rapidement une prise de conscience de l'existence de ce contre-modèle, comme dans cette Lettre pastorale datée du $1^{\text {er }}$ octobre 1686: « Mais ces habitans des montagnes [Cévennes] dès le mois de novembre suivant commencerent leurs assemblees secrettes. Et Dieu leur suscita du milieu d'eux des personnes qui sans études \& sans science se mirent à la tête de ces assemblées pour les édifier " (cité par Bost H., op.cit., p. 245. 
que cela n'était avant la révocation de l'édit de Nantes. Faut-il y voir la volonté d'une stricte application de la Discipline ecclésiastique dans la mesure où celle-ci prévoit l'organisation de jeûnes en cas de persécutions? Peut-être, mais pourquoi n'ont-ils pas été aussi réguliers entre 1661 et 1685 ? Il est probable que ce soit plutôt une particularité de cette " ecclésiologie de crise ». En reprenant les actes des synodes du Désert publiés par Edmond Hugues, on constate en effet cette présence quasi systématique de jeûnes dans les années 1718-1750, mais ce phénomène se poursuit au-delà de cette date, quelle que soit la province où se tiennent de telles assemblées ${ }^{48}$.

Dans les Cévennes, le règlement du synode provincial de 1730 prévoit même " qu'il sera chaque année célébré deux jeûnes dans nos églises, un dans le même temps où les églises de Suisse célèbrent le leur et l'autre dans le mois de février ${ }^{49}$ ". Le caractère exceptionnel de ces célébrations observé dans la période qui précède la révocation de l'édit de Nantes disparait, le jeûne devient un rituel commun de secours spirituel. Le synode national de cette même année 1730 décide cependant, en raison du danger qui menace les Églises du Désert, « de ne point fixer un jour dans un avenir assez éloigné, à cause que nos ennemis pourraient veiller avec beaucoup plus d'exactitude et par là même pouvoir nous causer du dommage ${ }^{50} »$; le synode national laisse aux synodes provinciaux le pouvoir de célébrer deux ou trois jeûnes par an, mais la date est laissée à la "prudence » des pasteurs et des prédicateurs « pour les faire célébrer dans le temps et le jour le plus convenable ${ }^{51}$ ». Le règlement fixé par le synode provincial du Bas-Languedoc en mai 1739 atteste également de cette évolution. Le chapitre 15 est justement consacré au jeûne ${ }^{52}$. Ainsi, " dans toutes les assemblées synodales, soit provinciales, soit nationales, on ordonnera un jeûne, même on en ordonnera plusieurs si le peuple se trouve dans quelque affliction, ou s'il est menacé » (article 1). Reprenant l'article de la Discipline ecclésiastique, le synode prévoit qu'un jeûne doit être également organisé « quand on voudra imposer les mains à quelque proposant ou le recevoir au saint ministère " (article 2), ou quand les consistoires procèderont à l'élection des

48. L'article 4 du synode provincial de Languedoc et Cévennes du 7 février 1718 donne en quelque sorte le ton: «En $4^{\mathrm{e}}$ lieu, soit à cause de la grande affliction où l'église est depuis de longues années, soit à cause des mœurs dépravées des peuples, la compagnie a trouvé bon d'ordonner un jour de jeûne pour tâcher en quelque manière d'apaiser le courroux du Seigneur justement allumé contre nous ». Il est suivi par le synode de Bas-Languedoc du 9 mai 1720, celui de Bas-Languedoc et Cévennes de $1721 \ldots$

49. Hugues E., op. cit., p. 93. Cette idée de célébrer un jeûne en même temps que les Églises réformées du Refuge se retrouve dans le synode de Haut-Languedoc de 1740 (art. 4) : «On s'humiliera extraordinairement devant Dieu pour un jeûne annuel le $11^{\mathrm{e}} \mathrm{d}^{\prime}$ octobre, si c'est un dimanche, ou le dimanche suivant, conformément à l'usage de nos frères réfugiés d'Angleterre et de Hollande qui célèbrent ce jour-là toutes les années en mémoire de la Révocation de l'édit de Nantes » (p. 164).

50. Ibid., p. 106.

51. Il est même précisé que " chaque synode national pourra en marquer un [jeûne] général, mais le jour inséré ne sera point inséré dans le règlement ".

52. Hugues E., op. cit., p. 373-374. 
anciens, "à l'exemple des Apôtres qui faisaient précéder ou suivre les élections des anciens et des ministres par des jeûnes et par des prières " (article 3). Le comportement des fidèles est même précisé: "Le véritable jeûne ne consiste pas à s'abstenir pendant quelques heures de nourriture ordinaire, mais principalement à s'abstenir du péché, à s'en dépouiller, et à se revêtir de la charité chrétienne qui se manifeste par la pratique de toutes sortes de bonnes œuvres » (article 4). C'est par le jeûne que les fidèles réformés peuvent participer dans de meilleures conditions aux œuvres.

Pour les années 1718-1750, Yves Krumenacker a relevé deux types de formules dans les synodes nationaux et provinciaux du Désert ${ }^{53}$ qui permettent de distinguer deux périodes différentes. La première qui s'achève vers 1745 montre un Dieu en colère contre des Églises réduites à un triste état ${ }^{54}$. La deuxième période correspond à la mise en lumière des causes de la colère divine, la corruption des Églises ${ }^{55}$. Outre les références traditionnelles à la " colère divine ", à la " désobéissance ", à la " corruption " des fidèles ou " aux mœurs dépravées des peuples », les articles évoquant le jeûne cherchent avant tout à unir la communauté dans ce que certains actes appellent " les tristes circonstances du temps », que ce soit " la grande affliction » dans laquelle se trouvent les Églises, ou selon les circonstances la préservation contre la peste ou que Dieu «fléchisse le cœur de nos schismatiques ». Cette volonté d'unité est d'autant plus importante que depuis 1685, le lieu qui permettait à la communauté de se retrouver a disparu. La célébration du jeûne doit donc montrer que la communauté est " un modèle de communauté confraternelle ${ }^{56}$ ». Ces thèmes de retrouvent dans les sermons prêchés au Désert. En 1738, Fléchier écrit par exemple:

« Nous avons le malheur d'être esclaves de ceux qui nous haïssent et d'estre méprisez de ceux qui ont vu notre opprobre, nos maisons d'oraisons sont dans la poussière depuis plus d'un demi siècle, nos frères gémissent dans les prisons et dans les galères. Pour tous ces maux, le colère du Seigneur n'est pas apaisée, mais sa main est encore estendue, nos ennemis forment tous les jours de nouveaux complots pour nous perdre et nous sommes exténués comme des brebis destinées à la boucherie ${ }^{57}$."

53. Krumenacker Y., "Jeûne et image de Dieu. Les synodes protestants du Désert ", Bulletin de la Société de l'Histoire du Protestantisme Français, 1994, p. 209-217.

54. Ce sont par exemple: " Pour apaiser le courroux de Dieu allumé contre nous ", " Pour fléchir la colère de Dieu justement allumée ", "Vu la désolation de nos églises ", " Nos églises étant toujours sous la croix, et la Providence continuant à nous éprouver depuis un siècle révolu »... Ibid., p. 211, note 8.

55. Les expressions relevées sont beaucoup plus nombreuses: «Vu la désolation accablante de l'église par les maux continuels qu'elle souffre ", "vu les violentes secousses des persécutions où les églises de ce royaume sont exposées, et la continuation de la guerre qui le désole ", "À cause de l'extrême corruption qui règne et qui va en augmentant, etvu que nos églises demeurent sous la croix $" .$. Ibid., p. 211-212, note 9.

56. Krumenacker Y., «L'élaboration d'un modèle protestant: les synodes du Désert ", Revue d'Histoire Moderne et Contemporaine, 42/1, janvier-mars 1995, p. 46-70.

57. Bibliothèque de la Société de l'Histoire du Protestantisme Français, ms 276, sermon prononcé le 12 août 1738 par Jean Molines, dit Fléchier. 
Il poursuit en expliquant que le fidèle cherche Dieu « avec succès par ces cinq choses: par la foi, par le jeûne et par la prière, et enfin par la conversion et la sainteté de la vie ${ }^{58}$ ».

Pour conclure, il faut souligner le rôle essentiel du jeûne dans les Églises réformées avant la Révocation comme élément d'unité de la communauté. Avec la révocation de l'édit de Nantes et les persécutions que subissent les communautés calvinistes, cette caractéristique ne fait que se renforcer. Toutefois, d'une pratique exceptionnelle avant 1685, la célébration du jeûne devient beaucoup plus régulière après 1715 , participant ainsi pleinement à la reconstruction des Églises réformées du Désert. Le jeûne apparaît enfin comme un élément de controverse dans le débat entre catholiques et protestants: derrière les attaques catholiques, c'est l'unité de la communauté qui est visée et son attitude politique à l'égard du souverain. Le jeûne est un argument pour remettre en cause le loyalisme des protestants. 